\title{
Universal Rapid Human Immunodeficiency Virus Screening at Delivery: A Cost-Effectiveness Analysis
}

\author{
Rachel K. Scott $\mathbb{D}^{1},{ }^{1,2}$ Stacia Crochet, ${ }^{3}$ and Chun-Chih Huang ${ }^{1,4}$ \\ ${ }^{1}$ MedStar Health Research Institute (MHRI), Washington, DC, USA \\ ${ }^{2}$ MedStar Washington Hospital Center (MWHC), Division of Women's and Infants' Services, Washington, DC, USA \\ ${ }^{3}$ Emory University Hospital, Atlanta, GA, USA \\ ${ }^{4}$ Georgetown-Howard Universities Center for Clinical and Translational Science, Washington, DC, USA
}

Correspondence should be addressed to Rachel K. Scott; rachelkscott@hotmail.com

Received 3 November 2017; Revised 19 January 2018; Accepted 4 February 2018; Published 14 March 2018

Academic Editor: Susan Cu-Uvin

Copyright (c) 2018 Rachel K. Scott et al. This is an open access article distributed under the Creative Commons Attribution License, which permits unrestricted use, distribution, and reproduction in any medium, provided the original work is properly cited.

\begin{abstract}
Objective. To determine the cost-effectiveness of universal maternal HIV screening at time of delivery to decrease mother-to-child transmission (MTCT), by comparing the cost and quality-adjusted life years (QALYs) of universal rapid HIV screening at time of delivery to two current standards of care for prenatal HIV screening in the United States. Study Design. We conducted a costeffectiveness analysis to compare the cost and QALY of universal intrapartum rapid HIV screening with two current standards of care: (I) opt-out rapid HIV testing limited to patients without previous third-trimester screening and (II) opt-out rapid HIV testing limited to patients without any prenatal screening. We developed a decision-tree model and performed sensitivity analyses to estimate the impact of variances in QALY, estimated lifetime medical costs, HIV prevalence, and cumulative incidence. Results. The incremental cost-effectiveness ratio for universal screening was $\$ 7,973.45 / \mathrm{QALY}$. The results remained robust to sensitivity analysis, except for annual cumulative incidence. In areas with an annual cumulative incidence rate of $<0.02 \%$ for reproductive-age women, the incremental cost-effectiveness ratio for the expanded program would exceed \$89,926.94/QALY, approaching the commonly applied cost-effectiveness thresholds (\$100,000/QALY). Conclusions. Intrapartum universal rapid HIV screening to decrease MTCT appears cost-effective in populations with high HIV incidence in the United States.
\end{abstract}

\section{Introduction}

Mother-to-child transmission (MTCT) of Human Immunodeficiency Virus (HIV) is largely preventable through early diagnosis and antiretroviral therapy (ART), including maternal and neonatal prophylaxis globally and cesarean delivery (CD) for high or unknown viral load in resourcerich settings. The risk of MTCT is significantly elevated among pregnancies with incident HIV infection compared to pregnancies with known maternal HIV [1]. In more than a quarter of cases of MTCT in the United States, maternal HIV infection is not diagnosed until after delivery $[2,3]$, obviating or delaying measures to prevent transmission. The Centers for Disease Control (CDC) and the American College of Obstetricians and Gynecologists (ACOG) both recommend HIV screening as early as possible in pregnancy and rapid screening in labor if patients have not had prior prenatal screening. In high risk patients, high incidence and prevalence communities, and medical centers with a screen-positive rate of greater than one per 1,000, ACOG recommends additional third-trimester screening prior to 36 weeks of gestation [2-5]. Based upon the CDC and ACOG recommendations, the standard of care in high prevalence communities is to perform a rapid HIV screen on patients in labor who have not had third-trimester screening and in low-incidence and low risk populations is to perform rapid screening in labor if there has been no previous HIV screening during pregnancy.

Most children born with perinatally acquired HIV (PAH) now survive to adulthood; however, not without both medical and behavioral health consequences [6-8]. In addition to the long-term effects of HIV infection, the adolescents and 
TABLE 1: Explanation of three strategies of care at delivery based on scenarios at prenatal care.

\begin{tabular}{lccc}
\hline Scenario & Universal screening & Standard of care I & Standard of care II \\
\hline $\begin{array}{l}\text { (A) HIV+ (identified during or before } \\
\text { prenatal HIV screening) }\end{array}$ & Treated per standard care for known HIV, no intrapartum rapid HIV screening \\
$\begin{array}{l}\text { (B) T1, T3 negative prenatal HIV screening } \\
\text { with negative result }\end{array}$ & Intrapartum rapid HIV screening & Usual care & Usual care \\
$\begin{array}{l}\text { (C) T1 negative prenatal HIV screening (no } \\
\text { T3 screening) }\end{array}$ & Intrapartum rapid HIV screening & Performing rapid screening \\
$\begin{array}{l}\text { (D) T3 negative prenatal HIV screening } \\
\text { with negative result (no T1 screening) }\end{array}$ & Intrapartum rapid HIV screening & Usual care \\
(E) No T1 and T3 prenatal HIV screening & Intrapartum rapid HIV screening & Intrapartum rapid HIV screening & Intrapartum rapid \\
\hline
\end{tabular}

$\mathrm{T} 1$, the first trimester; $\mathrm{T} 3$, the third trimester.

young adults who have grown up with $\mathrm{PAH}$ frequently have virologic resistance from inconsistent long-term ART use. Additionally, this patient population has demonstrated increased high risk sexual behavior, mental illness, and substance abuse [7, 9, 10]. From birth, these individuals face a lifetime of chronic disease, as well as the associated stigma and resulting behavioral consequences and health comorbidities.

We hypothesized that universal, opt-out, rapid HIV screening upon admission for delivery (either vaginal or CD) to Labor and Delivery (L\&D) provides an additional opportunity, in high incidence and prevalence populations, to prevent MTCT in mothers who were not previously diagnosed with HIV, secondary to either lack of prenatal screening or seroconversion in the third trimester of pregnancy. Diagnosis and treatment of intrapartum HIV cannot prevent MTCT that may have already occurred in utero; however maternal intravenous zidovudine (AZT) prophylaxis, CD before active labor or rupture of membranes (ROM), and enhanced neonatal antiretroviral prophylaxis with 2 or 3 ART all independently decrease the risk of intrapartum MTCT [11-14]. Recognizing the logistical challenge of intrapartum AZT prophylaxis due to the time limitation of active labor and the unclear benefit of CD during active labor or after ROM, at minimum, the diagnosis of HIV in labor allows for immediate neonatal prophylaxis postpartum, which alone has been demonstrated to decrease perinatal transmission to $2.2-2.4 \%$ [12] (from $25.5 \%$ without intervention [15]), the opportunity to counsel against breastfeeding, and early diagnosis and treatment of neonatal HIV.

The prevalence of HIV among women in the District of Columbia (DC) is over eightfold higher than the national average (1.4\% compared to $0.17 \%$ nationally) [16]. The DC Department of Health estimates the prevalence to be even higher among reproductive aged women. Given the high prevalence $(1.9 \%)$ and annual incidence $(0.087 \%)$ among reproductive-age women [17], our medical center recently implemented universal rapid HIV screening of all patients with viable pregnancies admitted for delivery. This study evaluated the cost-effectiveness of universal rapid HIV screening at time of delivery in a high HIV prevalence and incidence area, such as DC.

\section{Methods}

2.1. Study Model. We set out to test our hypothesis that universal rapid HIV screening would be cost-effective in high incidence and prevalence areas of the United States, secondary to identification of previously undiagnosed maternal $\mathrm{HIV}$ and resulting in timely intrapartum and postpartum interventions to prevent MTCT and improve neonatal outcomes. We conducted a cost-effectiveness analysis from the societal perspective to compare cost and QALY of our optout intrapartum universal rapid HIV screening strategy with the two current alternative standards of care: standard of care I, opt-out rapid HIV screening limited to patients without previous third-trimester HIV screening; standard of care II, opt-out rapid HIV screening limited to patients without any prenatal HIV screening.

After Institutional Review Board approval from the MedStar Health Research Institute (\#2015-069), we developed a decision-tree model to analyze the cost-effectiveness of universal, opt-out, rapid HIV screening of pregnant patients admitted for delivery (spontaneous labor or scheduled induction of labor or cesarean delivery), taking into account the incremental lifetime costs and the loss of QALY associated with MTCT and PAH. We designed our model using the estimated values of parameters listed in Table 2. In our decisiontree model, we took into account the different potential combinations of prenatal HIV screening algorithms, adherence to screening algorithms, and screening results. We used the incidence and prevalence of reproductive aged women HIV in DC to derive the probability maternal HIV acquisition and seroconversion between prenatal HIV screening and intrapartum HIV screening. Taking into consideration the spectrum of clinical presentations to labor and delivery, we then applied the different intrapartum screening strategies to potential clinical scenarios (universal rapid HIV screening, standard of care I, and standard of care II) (Table 1). Using the estimated maternal intrapartum rapid HIV results/maternal intrapartum HIV status, based on the intrapartum screening strategies, we projected the different risks of MTCT for each strategy and accordingly the risk of a HIV exposed versus a HIV infected neonate. From our model's findings, for each screening strategy, we then calculated the estimated additional cost and reduced QALYs for an infected neonate. 
TABLE 3: Sensitivity analysis values and ranges.

\begin{tabular}{lcc}
\hline Variable & Range & Reference \\
\hline QALY saved per prevention of 1 case of MTCT & $5-25^{\top}$ & {$[20,25-30]$} \\
Estimated lifetime medical cost of PAH & $\$ 250 \mathrm{k}-\$ 550 \mathrm{k}]$ & {$[20,25-28,31,32]$} \\
HIV prevalence & $0.5 \%-10 \%$ & $\ddagger$ \\
HIV annual cumulative incidence & $0.01 \%-0.15 \%$ & $\$$ \\
Cost of rapid test & $\$ 11.90-\$ 16.32$ & $\$ 185-\$ 210$ \\
Neonatal prophylaxis & $\$ 185-$ & $€$ \\
\hline
\end{tabular}

${ }^{9}$ Range was set to cover the cost value obtained from the references (after it was updated to 2015 dollars). ${ }^{7} \mathrm{~A} 3 \%$ discount rate was applied to indicate a present value. ${ }^{\ddagger}$ The range was set to examine the impact of extreme value on the estimated outcomes, not necessarily indicating a highest or lowest rate in District of Columbia. ${ }^{-1}$ Range was set based on the minimum and maximum of Medicare Clinical Diagnostic Laboratory Fee Schedule among different locations for the same service. ${ }^{\epsilon}$ We also considered three-drug prophylaxis, with Lamivudine in addition to AZT and Nevirapine. $240 \mathrm{ml}$ of the $10 \mathrm{mg} / \mathrm{ml}$ oral solution costs $\$ 76.52$, per the Neonatal Pharmacy Department of MedStar Washington Hospital Center. By the US AIDS info guidelines, a $3500 \mathrm{~g}$ neonate requires $784 \mathrm{mg}$ total of Lamivudine (\$25), in addition to AZT and Nevirapine (\$185).

HIV test in our model, we used the proportion of CD found by Bulterys et al. to reflect clinical practice, regardless of the unknown protective effect [14].

Regardless of the HIV screening strategy, patients with known HIV would have been treated per standard of care (with maternal ART, maternal prophylaxis and CD as indicated, and neonatal prophylaxis) and therefore would not undergo intrapartum universal rapid HIV screening and would have no impact on the incremental cost and effectiveness of our analysis. Figure 1 is a simplified depiction of decision-tree used for the analysis.

2.2. Parameters. The measures of costs included the materials and services of the rapid HIV test, associated treatments for patients who tested positive, additional costs of $\mathrm{CD}$, and the lifetime medical costs for a newborn with PAH. We assumed that other costs were the same across the three strategies and were not included in the cost analysis. We adjusted all monetary values to the 2015 USD. We applied a 3\% discount rate to estimate the savings in both QALY and lifetime medical costs in present values by preventing one case of PAH. Detailed values for each input parameter are listed in Table 2. We obtained these values and associated assumptions from a combination of previously published peer reviewed medical literature and statistics from the Washington DC Department of Health.

2.3. Cost-Effectiveness Analysis. We used the incremental cost-effectiveness ratio (ICER), defined as the incremental cost divided by incremental health gains, for the costeffectiveness analysis. We estimated the incremental cost per QALY of universal rapid HIV screening, using standard of care I and standard of care II as the references, respectively. $\$ 50,000$ to $\$ 100,000$ per QALY is considered the standard acceptable range for a cost-effective intervention [22, 23]; however, Ubel and colleagues and others argue that this range is overly conservative and that even the upper threshold of $\$ 100,000$ is still a too low value to put on life [24]. In our study, we used the upper limit of the conservative estimate, $\$ 100,000$, as the threshold for a cost-effective intervention.

2.4. Sensitivity Analysis. For further comparison between the proposed strategy (universal rapid HIV screening) and both the standard of care in the DC area (standard of care I) and standard of care II, we conducted univariate sensitivity analyses to estimate the impact of selected variables on the model, including HIV prevalence and cumulative incidence, QALY saved by preventing one case of MTCT, and lifetime medical cost of treatment for a neonate with $\mathrm{PAH}$. The range for each parameter incorporates the highest and lowest values from the medical literature or expert opinions. Considering the argument that a higher lifetime medical cost in treating HIV leads to a lower level of difference in QALY, compared to a HIV negative individual, we also conducted a two-way sensitivity analysis by varying the cost and the difference in QALY. Table 3 shows the range of variation of parameters for these variables.

\section{Results}

Among the three strategies, the universal screening appeared to have the highest estimated cost and QALYs per 10,000 pregnant women (Table 4). Using standard of care I as the reference strategy, the incremental cost of the universal screening per 10,000 patients was $\$ 39,827.83$ with 5.01 QALYs saved. The ICER was estimated to be $\$ 7,943.45$. Using standard of care II as the reference, the ICER for the universal screening was $\$ 3,610.58$. Both estimates are less than $\$ 100,000-$ a conservative threshold for a cost-effective intervention. Standard of care I achieved higher QALYs than standard of care II with lower estimated cost, indicating that standard of care II is inferior.

Table 5 shows the results of the sensitivity analyses for the ICER of universal rapid HIV screening to standard of care I. For the QALY saved, lifetime medical costs, rapid test cost, cost of neonatal prophylaxis, and HIV prevalence, the calculated ICERs were below the threshold for the extreme values of range, indicating that the results remained robust. We obtained similar results in second sensitivity analyses comparing universal rapid HIV screening to standard of care II (Table 6).

Figures 2 and 3 illustrate the sensitivity analyses for the ICER of universal screening relative to standard of care in the DC area. As demonstrated in Figure 2, favorable ICER was dependent upon a high cumulative incidence of HIV. 


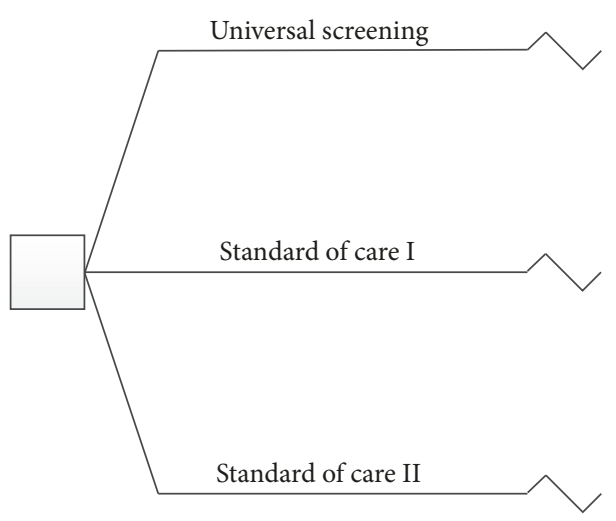

(a)

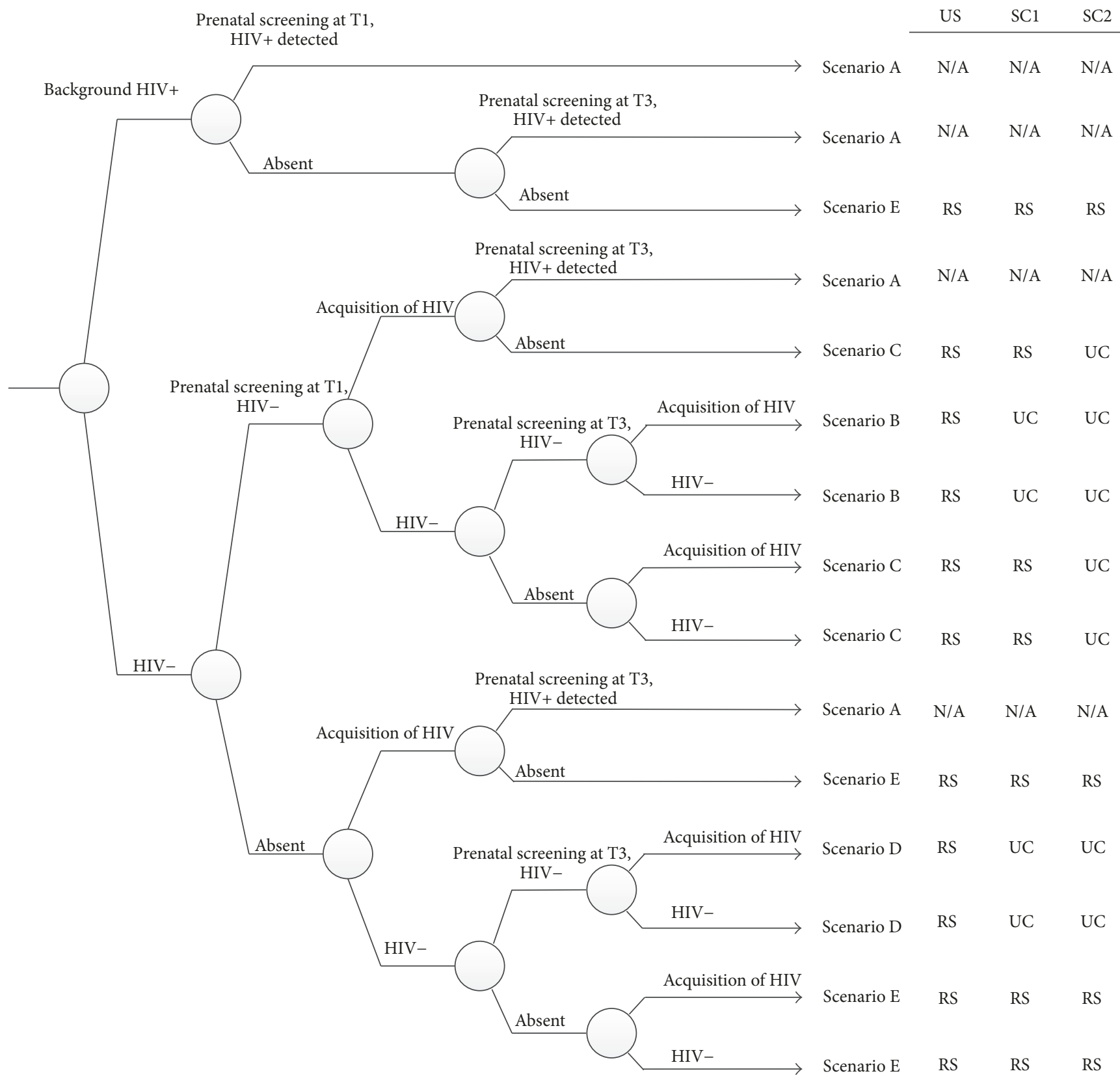

(b)

Figure 1: Continued. 


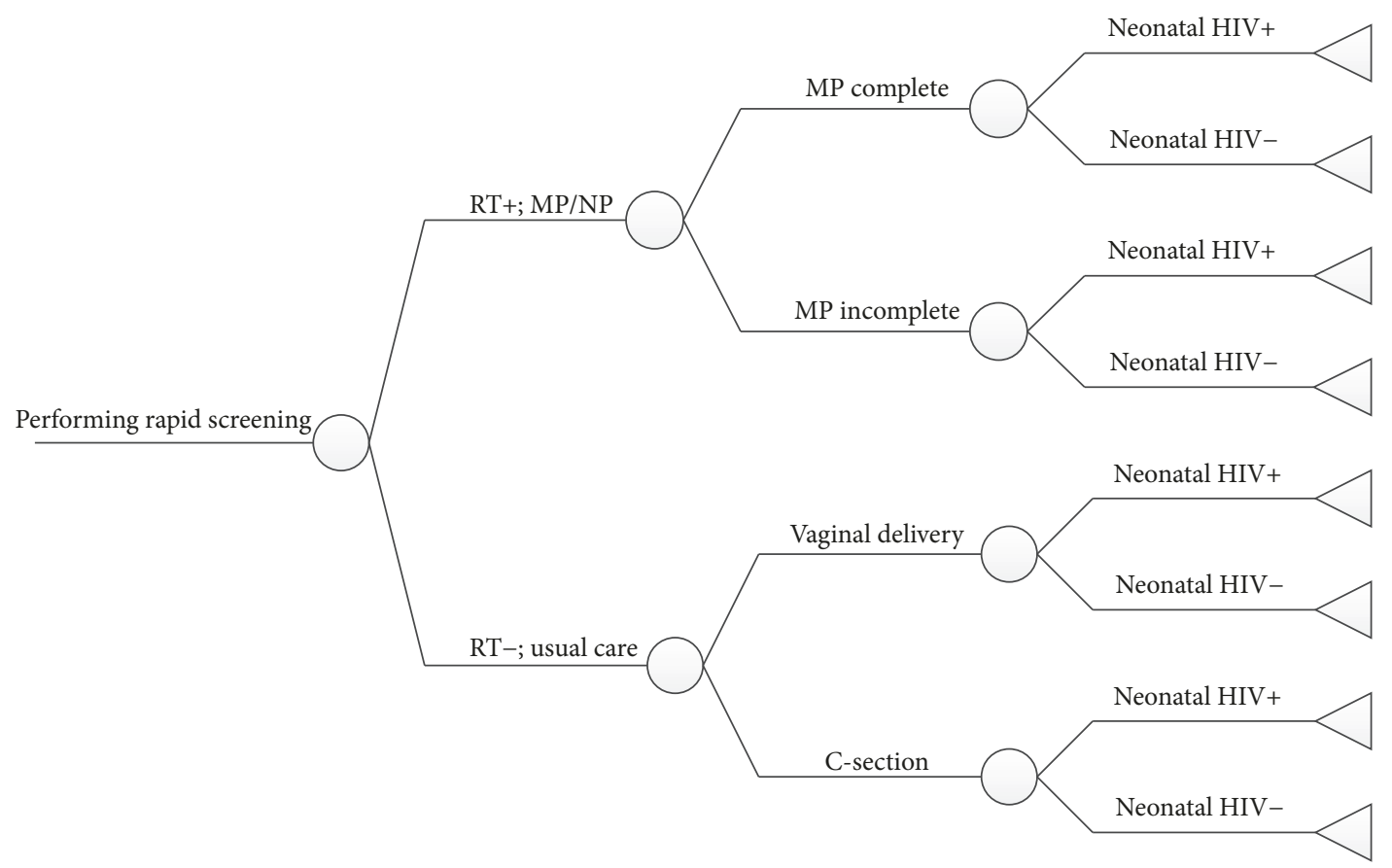

(c)

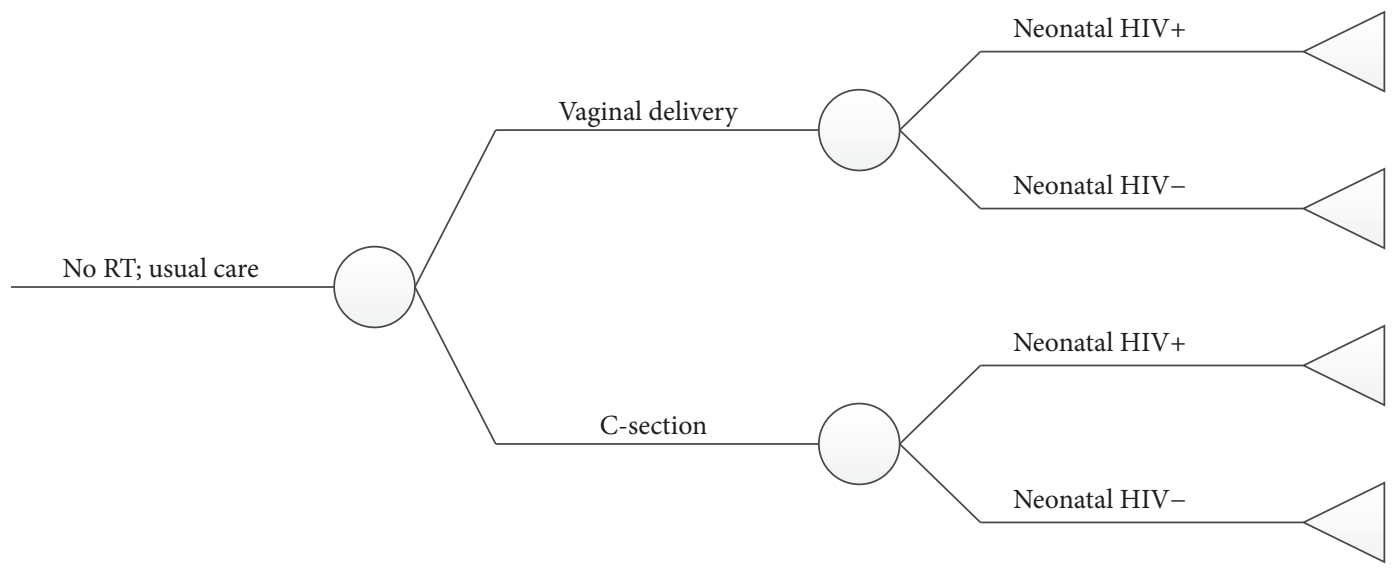

(d)

FIGURE 1: Simplified depiction of decision-tree used for the analysis. (a) Three strategies, universal rapid HIV screening, standard of care I, and standard of care II, are compared in the analysis. (b) Scenarios based on prenatal HIV screening and the screening result. (For simplicity, the figure assumes $100 \%$ sensitivity and $100 \%$ specificity for the prenatal HIV screening.) (c) The care pattern for patients undergoing intrapartum rapid screening. (d) The care pattern for patients not undergoing intrapartum rapid screening. US: universal screening; SC1, standard of care I; SC2, standard of care II; RS, rapid screen; UC, usual care; MP, maternal prophylaxis; NP, neonatal prophylaxis; T1, first trimester; T3, third trimester.

Specifically, if the annual cumulative incidence rate were less than $0.02 \%$ (i.e., less than a fifth of the rate in DC) the incremental cost-effectiveness ratio for the expanded program would exceed $\$ 89,926.94 /$ QALY, approaching the cost-effectiveness threshold (\$100,000/QALY). When the incidence rate approaches $0.13 \%$ (i.e., about 1.5 times in DC), the universal screening is both less expensive and more effective screening strategy.

Figure 3 shows the combined impact of the QALYs saved from averting one case of MTCT and the lifetime medical cost of PAH on the estimate of ICER, based on a two-way sensitivity analysis. In the direction of inverse relationship between the QALYs saved and the lifetime medical cost $(\mathrm{A} \leftrightarrow$ B), the scenario with low cost and large QALYs saved (point B) has higher ICER compared to the one with high cost and small QALYs saved (point A). However, the universal rapid HIV testing still demonstrated a cost-effective option $(\$ 8,762.90 /$ QALY; point B). The analysis further indicated that low lifetime medical cost and low incremental QALY lead to high ICER. However, even using the highest estimate of 


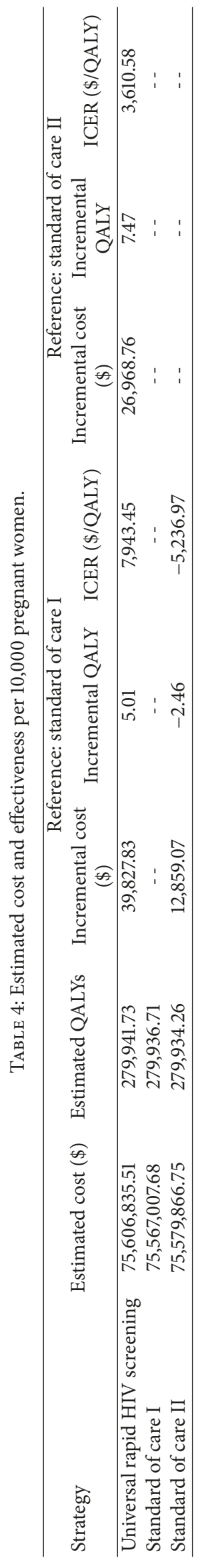


TABLE 5: Univariate sensitivity analysis of universal rapid HIV screening relative to standard of care I.

\begin{tabular}{lcccc}
\hline Variable & Detected range & Estimated incremental cost $(\$)$ & $\begin{array}{c}\text { Estimated } \\
\text { incremental QALY }\end{array}$ & $\begin{array}{c}\text { Incremental } \\
\text { cost-effectiveness ratio } \\
\text { (\$/QALY) }\end{array}$ \\
\hline $\begin{array}{l}\text { QALY per prevention of 1 case of } \\
\text { MTCT }\end{array}$ & $5 \sim 25$ & $39,827.83$ & $1.32 \sim 6.60$ & $30,185.10 \sim 6,037.02$ \\
$\begin{array}{l}\text { Estimated lifetime medical cost } \\
\text { of PAH }\end{array}$ & $\$ 250 \mathrm{k} \sim \$ 550 \mathrm{k}$ & $57,811.19 \sim(-21,356.02)$ & 5.01 & $11,530.13 \sim(-4,259.34)$ \\
HIV prevalence & $0.5 \% \sim 10 \%$ & $40,396.22 \sim 36,539.30$ & $5.09 \sim 4.60$ & $7,943.45 \sim 7,943.45$ \\
$\begin{array}{l}\text { HIV annual cumulative } \\
\text { incidence }\end{array}$ & $0.01 \% \sim 0.15 \%$ & $113,233.84 \sim(-20,316.76)$ & $0.58 \sim 8.65$ & $196,399.01 \sim(-2,348.85)$ \\
$\begin{array}{l}\text { Cost of rapid test } \\
\text { Cost of neonatal prophylaxis }\end{array}$ & $\$ 11.90 \sim \$ 16.32$ & $15,661.65 \sim 50,341.69$ & 5.01 & $3,123.63 \sim 10,040.38$ \\
\end{tabular}

TABLE 6: Univariate sensitivity analysis of universal rapid HIV screening relative to standard of care II.

\begin{tabular}{lcccc}
\hline Variable & Detected range & $\begin{array}{c}\text { Estimated incremental } \\
\text { cost }(\$)\end{array}$ & $\begin{array}{c}\text { Estimated } \\
\text { incremental QALY } \\
\left(10^{-5}\right)\end{array}$ & $\begin{array}{c}\text { Incremental cost-effectiveness } \\
\text { ratio }(\$ / Q A L Y)\end{array}$ \\
\hline $\begin{array}{l}\text { QALY saved per prevention of } 1 \\
\text { case of MTCT }\end{array}$ & $5 \sim 25$ & $26,968.76$ & $1.97 \sim 9.83$ & $13,720.22 \sim 2,744.04$ \\
$\begin{array}{l}\text { Estimated lifetime medical cost } \\
\text { of PAH }\end{array}$ & $\$ 250 \mathrm{k} \sim 550 \mathrm{k}$ & $53,759.00 \sim(-64,178.29)$ & 7.47 & $7,197.27 \sim(-8,592.20)$ \\
$\begin{array}{l}\text { HIV prevalence } \\
\text { HIV annual cumulative }\end{array}$ & $0.5 \% \sim 10 \%$ & $27,353.64 \sim 24,741.98$ & $7.58 \sim 6.85$ & $3,610.58 \sim 3,610.58$ \\
$\begin{array}{l}\text { incidence } \\
\text { Cost of rapid test }\end{array}$ & $0.01 \% \sim 0.15 \%$ & $136,311.47 \sim(-62,625.81)$ & $0.86 \sim 12.89$ & $158,710.06 \sim(-4,860.01)$ \\
Cost of neonatal prophylaxis & $\$ 11.90 \sim \$ 16.32$ & $(-2,660.25) \sim 39,859.31$ & 7.47 & $(-356.16) \sim 5,336.37$ \\
\end{tabular}

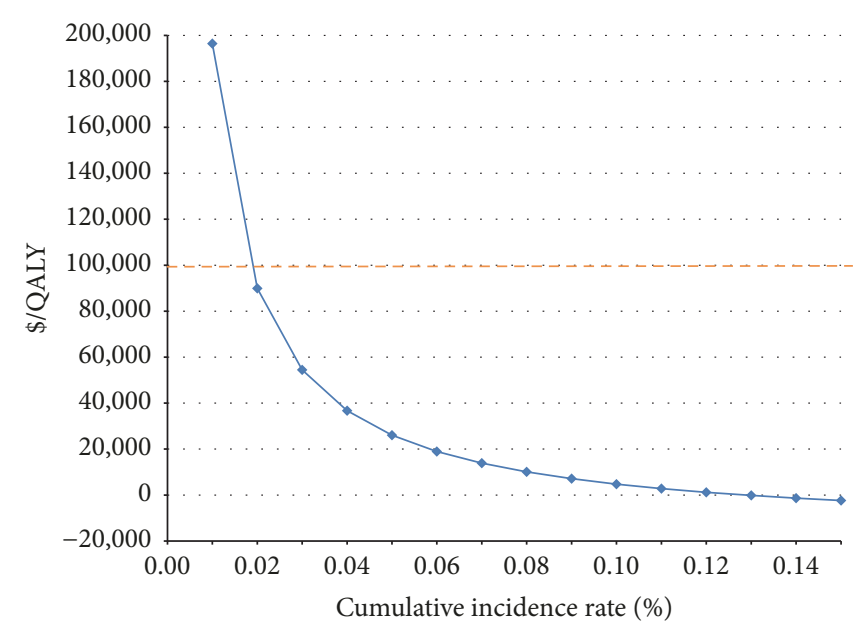

FIGURE 2: One-way sensitivity analysis: impact of annual cumulative incidence on ICER (universal screening relative to standard of care in the DC area).

\$250k lifetime medical costs and the lowest level of QALY averted (5), the ICER remained below the $\$ 100,000$ threshold $(\$ 43,814.50 / \mathrm{QALY}$; point C).

\section{Discussion}

Diagnosis and treatment of maternal HIV is crucial to eliminating MTCT and the eventual eradication of HIV. In our model, we considered the costs of the rapid HIV test and of the preventive interventions, taking into account sensitivity and specificity of the rapid HIV test at time of delivery and of previous prenatal screening, along with the probability of having had previous prenatal screening.

Our sensitivity analysis compared universal rapid HIV screening to "standard of care I," and as we hypothesized we found universal rapid HIV screening to be more cost-effective in areas with an annual cumulative incidence of HIV in women of reproductive age greater than $0.02 \%$. In areas with a very low cumulative incidence of HIV, specifically less than $0.02 \%$, universal rapid HIV screening will be of questionable cost-effectiveness. Using the threshold of $\$ 100,000$ for a costeffective intervention, "standard of care I" was only more favorable than universal rapid HIV screening or "standard of care II" if the annual cumulative incidence was less than

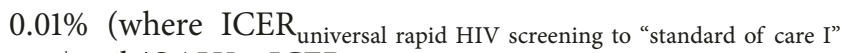
$=\$ 196 \mathrm{k} / \mathrm{QALY} ; \quad$ ICER "standard of care I" to "standard of care II" = $\$ 82 \mathrm{k} / \mathrm{QALY})$. To expand our model, we varied the values of $\mathrm{HIV}$ prevalence and incidence to assess whether the universal rapid HIV screening would still be the superior strategy 


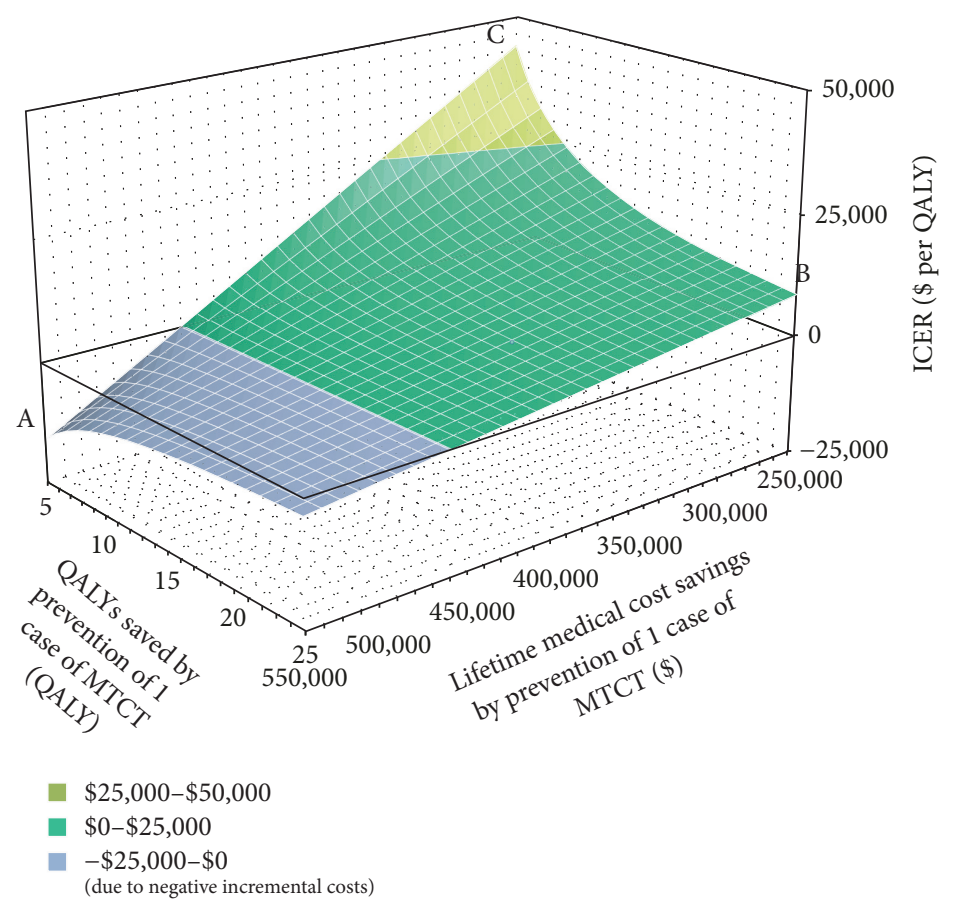

FIgURE 3: Two-way sensitivity analysis: impact of QALY saved per prevented case of MTCT and lifetime medical costs of PAH on ICER (universal screening relative to standard of care in the DC area).

in different communities. We found that HIV prevalence was not the key driver in our sensitivity analysis, but rather cumulative incidence. The cumulative incidence highly affected the estimated ICER; specifically lower cumulative incidence resulted in higher ICER. The decreased impact of HIV prevalence in our sensitivity analysis was secondary to the high sensitivity of prenatal HIV screening (100\% in our assumption); pregnant women with known HIV would be identified in the prenatal period and treated per guidelines, as discussed previously, leading to a limited impact in our model.

This model can be applied to other communities to determine whether intrapartum universal rapid HIV screening is cost-effective for their patient population, depending upon their local HIV incidence and the costs of interventions to test and prevent MTCT. Within the United States, universal rapid HIV screening would likely be cost-effective and should be considered in other high incidence areas such as Baltimore, Miami, New Orleans, and New York City. By the substituting local costs (such as the cost of maternal AZT) and local probabilities (such as maternal cumulative incidence in the community and CD rate), communities can individualize this model to gauge the cost-effectiveness of universal rapid HIV screening at time of delivery. We are currently developing a computer and mobile device based application to allow adaptation of the model to other communities.

The model presented in this paper is a simplified representation of a complicated medical and psychosocial scenario. For example, in patients who arrive in active labor or after rupture of membranes, there is limited literature on the benefit of CD and we assumed no protective effect in our model. Our model used the published experience of other centers to provide the most accurate real-world model we could create. Our model focuses on the health outcomes of HIV exposed neonates at high risk of MTCT and the economic burden of their medical care. We chose QALY as the basis for our model, which takes into account both healthrelated quality of life and adjusted life-expectancy [24]. Any model is limited in its ability to take into account all of the medical, economic, and psychosocial components at play in real life. We reviewed and considered multiple additional outcome measures that were not ultimately included in our model, such as maternal benefits and burdens related to universal rapid HIV screening at time of delivery, the lifetime nonmedical costs (such as loss of productivity) or the other opportunity costs affecting social activities due to the HIV, potential benefits to the health care institutions that adopt universal rapid HIV screening, such as potential savings from an avoided lawsuit, and other potential reduction of societal burdens, including risk of transmission to other individuals by a PAH patient during lifetime. While we recognize the inherent simplification and limitations of our model, we have incorporated the major driving medical and economic factors in our analysis.

Improvement in medical technology has facilitated better treatment and outcomes for individuals living with HIV, resulting in longer life-expectancy with better quality of life. The gap between the levels of QALY for a HIV infected neonate and an uninfected neonate has been decreasing and, by living longer, the lifetime medical cost of treating $\mathrm{PAH}$ is 
inevitably increasing. Even with the most extreme scenario using the lowest QALYs saved and the highest lifetime medical cost obtained from our references for the two-way sensitivity analysis, the ICER of universal rapid HIV screening to standard of care I is still below the $\$ 100,000 /$ QALY threshold, indicating the impact of the potential adverse association between the QALYs saved and the lifetime medical cost on the decision is limited. That said, we acknowledge the possible underestimate of ICERs in our study.

Our model does not involve complicative logic, such as repeated cycles, event tracking, or stage transition. Although we considered both static decision-tree and microsimulation modeling, we opted for the decision-tree, as we did not find that microsimulation conferred a more in-depth understanding of cost-effectiveness of universal intrapartum HIV screening.

\section{Conclusion}

We developed a novel model to test the cost-effectiveness of HIV screening protocols in pregnancy and those intrapartum. Through our model, we justify opt-out universal rapid HIV screening at delivery to be a highly cost-effective strategy for preventing MTCT in high incidence areas.

\section{Additional Points}

Condensation. Universal rapid HIV screening at time of delivery to decrease mother-to-child transmission appears cost-effective in populations with high HIV incidence in the United States.

\section{Disclosure}

This research was presented on July 21 at the 21st International AIDS Conference (AIDS 2016) in Durban, South Africa (July $18-22)$.

\section{Conflicts of Interest}

The authors report no conflicts of interest.

\section{Acknowledgments}

This project was funded in part by Federal Funds (Grant no. UL1TR000101 previously UL1RR031975) from the National Center for Advancing Translational Sciences (NCATS), National Institutes of Health (NIH), through the Clinical and Translational Science Awards (CTSA) Program, a trademark of DHHS, part of the Roadmap Initiative, "Re-Engineering the Clinical Research Enterprise."

\section{References}

[1] A. L. Drake, A. Wagner, B. Richardson, and G. John-Stewart, "Incident HIV during pregnancy and postpartum and risk of mother-to-child HIV transmission: a systematic review and meta-analysis," PLoS Medicine, vol. 11, no. 2, Article ID e1001608, 2014.
[2] A. F. Camacho-Gonzalez, M.-H. Kingbo, A. Boylan, A. R. Eckard, A. Chahroudi, and R. Chakraborty, "Missed opportunities for prevention of mother-to-child transmission in the United States," AIDS, vol. 29, no. 12, pp. 1511-1515, 2015.

[3] CDC, "Achievements in public health: reduction in perinatal transmission of HIV infection-United States, 1985-2005," Morbidity and Mortality Weekly Report (MMWR), pp. 592-597, 2006.

[4] ACOG, "Prenatal and perinatal human immunodeficiency virus testing: expanded recommendations," Committee Opinion, 2015.

[5] B. M. Branson, H. H. Handsfield, M. A. Lampe, R. S. Janssen, A. W. Taylor, and S. B. Lyss, "Revised recommendations for HIV testing of adults, adolescents, and pregnant women in healthcare settings," in MMWR Recommendations and Reports, vol. 55, pp. 1-17, Centers for Disease Control and Prevention (CDC), 2006.

[6] R. Hazra, G. K. Siberry, and L. M. Mofenson, "Growing up with HIV: Children, adolescents, and young adults with perinatally acquired HIV infection," Annual Review of Medicine, vol. 61, pp. 169-185, 2010.

[7] L. J. Koenig, S. Nesheim, and S. Abramowitz, "Adolescents with perinatally acquired HIV: emerging behavioral and health needs for long-term survivors," Current Opinion in Obstetrics and Gynecology, vol. 23, no. 5, pp. 321-327, 2011.

[8] C. A. Mellins, K. Tassiopoulos, K. Malee et al., "Behavioral health risks in perinatally HIV-exposed youth: co-occurrence of sexual and drug use behavior, mental health problems, and nonadherence to antiretroviral treatment," AIDS Patient Care and STDs, vol. 25, no. 7, pp. 413-422, 2011.

[9] K. Tassiopoulos, A.-B. Moscicki, C. Mellins et al., "Sexual risk behavior among youth with perinatal HIV infection in the United States: predictors and implications for intervention development," Clinical Infectious Diseases, vol. 56, no. 2, pp. 283-290, 2013.

[10] S. Kapetanovic, R. E. Wiegand, K. Dominguez et al., "Associations of medically documented psychiatric diagnoses and risky health behaviors in highly active antiretroviral therapyexperienced perinatally HIV-infected youth," AIDS Patient Care and STDs, vol. 25, no. 8, pp. 493-501, 2011.

[11] The International Perinatal HIV Group, "The mode of delivery and the risk of vertical transmission of human immunodeficiency virus type $1-$ A meta-analysis of 15 prospective cohort studies," The New England Journal of Medicine, vol. 340, no. 13, pp. 977-987, 1999.

[12] K. Nielsen-Saines, D. H. Watts, V. G. Veloso et al., “Three postpartum antiretroviral regimens to prevent intrapartum HIV infection," The New England Journal of Medicine, vol. 366, no. 25, pp. 2368-2379, 2012.

[13] Panel on Treatment of Pregnant Women with HIV Infection and Prevention of Perinatal Transmission. Recommendations for Use of Antiretroviral Drugs in Transmission in the United States, http:// aidsinfo.nih.gov/contentfiles/lvguidelines/PerinatalGL.pdf, 2018.

[14] M. Bulterys, D. J. Jamieson, M. J. O’Sullivan et al., "Rapid HIV1 testing during labor: a multicenter study," The Journal of the American Medical Association, vol. 292, no. 2, pp. 219-223, 2004.

[15] E. M. Connor, R. S. Sperling, R. Gelber et al., "Reduction of maternal-infant transmission of human immunodeficiency virus type 1 with zidovudine treatment," The New England Journal of Medicine, vol. 331, no. 18, pp. 1173-1180, 1994.

[16] CDC. National Center for HIV/AIDS, Viral Hepatitis, STD, and TB Prevention Atlas. https://www.cdc.gov/nchhstp/. 
[17] Data were based on women between ages of 14-45 at the end of 2012, obtained/derived from Department of Health, Government of the District of Columbia.

[18] Bayer Health Care LLC. ADVIA Centaur Assay Manual: HIV 1/O/2 Enhanced (EHIV) Assay for the Detection of Antibodies to Human Immunodeficiency Virus Type 1, Including Group O (HIV-1 + “O”) and/or Type 2 (HIV-2), http://www.fda.gov/downloads/ucm091286.pdf, 2006.

[19] A. W. Helfgott, B. Castner, M. Bates, D. Mayne, P. NicholsonKuenn, and A. Thorp J, "Rapid human immunodeficiency virus-1 testing to prevent perinatal HIV," Obstetrics \& Gynecology, vol. 107, no. 4, pp. 2S-3S, 2006.

[20] Pediatric HIV Surveillance, Centers for Disease Control and Prevention, National Center for HIV Viral Hepatitis STD and TB Prevention, 2012, http://www.cdc.gov/hiv/pdf/statistics_surveillance_Pediatric.pdf.

[21] C. J. McCabe, S. J. Goldie, and D. N. Fisman, "The cost-effectiveness of directly observed highly-active antiretroviral therapy in the third trimester in HIV-infected pregnant women," PLoS ONE, vol. 5, no. 4, Article ID e10154, 2010.

[22] T. Shiroiwa, Y.-K. Sung, T. Fukuda, H.-C. Lang, S.-C. Bae, and K. Tsutani, "International survey on willingness-to-pay (WTP) for one additional qaly gained: What is the threshold of cost effectiveness?" Health Economics, vol. 19, no. 4, pp. 422-437, 2010.

[23] H. Hyewon and M. Levine, "Determing the Threshold of Acceptability of an ICER when Natural Health Units are used," Journal of Population Therapeutics and Clinical Pharmacology, vol. 19, p. 234, 2012.

[24] P. A. Ubel, R. A. Hirth, M. E. Chernew, and A. M. Fendrick, "What is the price of life and why doesn't it increase at the rate of inflation?" JAMA Internal Medicine, vol. 163, no. 14, pp. 16371641, 2003.

[25] F. Sassi, "Calculating QALYs, comparing QALY and DALY calculations," Health Policy and Planning, vol. 21, no. 5, pp. 402408, 2006.

[26] D. R. Holtgrave and S. D. Pinkerton, "Updates of cost of illness and quality of life estimates for use in economic evaluations of HIV prevention programs," Journal of Acquired Immune Deficiency Syndromes, vol. 16, no. 1, pp. 54-62, 1997.

[27] J. M. Mrus, S. J. Goldie, M. C. Weinstein, and J. Tsevat, "The cost-effectiveness of elective Cesarean delivery for HIV-infected women with detectable HIV RNA during pregnancy," AIDS, vol. 14, no. 16, pp. 2543-2552, 2000.

[28] P. G. Farnham, C. Gopalappa, S. L. Sansom et al., "Updates of lifetime costs of care and quality-of-life estimates for HIVinfected persons in the United States: Late Versus early diagnosis and entry into care," Journal of Acquired Immune Deficiency Syndromes, vol. 64, no. 2, pp. 183-189, 2013.

[29] P. G. Farnham, D. R. Holtgrave, C. Gopalappa, A. B. Hutchinson, and S. L. Sansom, "Lifetime costs and quality-adjusted life years saved from HIV prevention in the test and treat era," Journal of Acquired Immune Deficiency Syndromes, vol. 64, no. 2, pp. e15-e18, 2013.

[30] A. B. Hutchinson, P. Patel, S. L. Sansom et al., "Cost-effectiveness of pooled nucleic acid amplification testing for acute HIV infection after third-generation HIV antibody screening and rapid testing in the United States: A comparison of three public health settings," PLoS Medicine, vol. 7, article e1000342, no. 9, 2010.

[31] D. R. Holtgrave, R. J. Wolitski, S. L. Pals et al., "Cost-utility analysis of the housing and health intervention for homeless and unstably housed persons living with HIV," AIDS and Behavior, vol. 17, no. 5, pp. 1626-1631, 2013.

[32] B. R. Schackman, K. A. Gebo, R. P. Walensky et al., "The lifetime cost of current human immunodeficiency virus care in the United States," Medical Care, vol. 44, no. 11, pp. 990-997, 2006. 


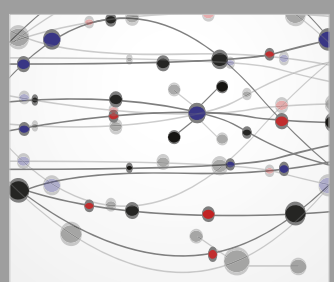

The Scientific World Journal
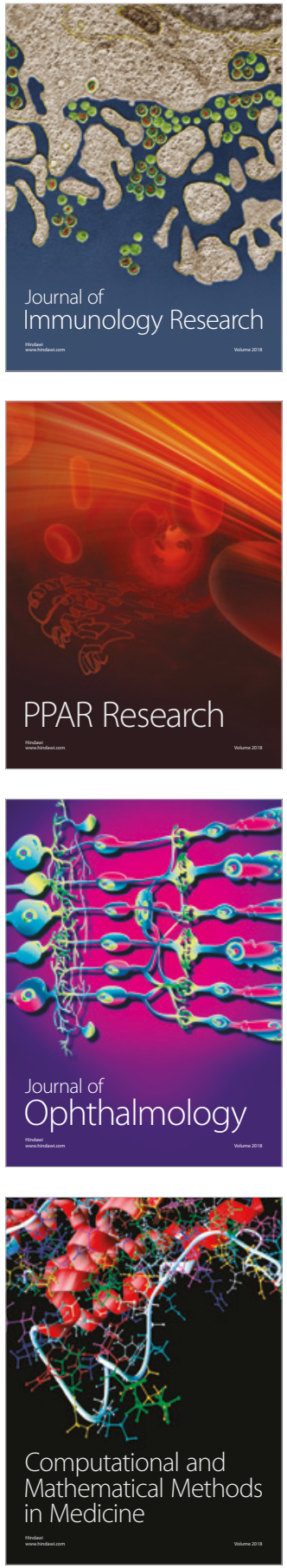

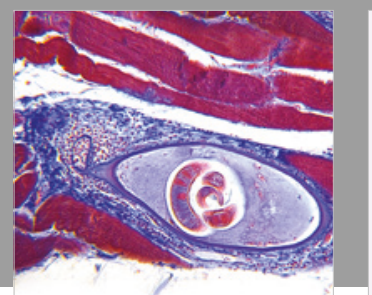

Gastroenterology Research and Practice

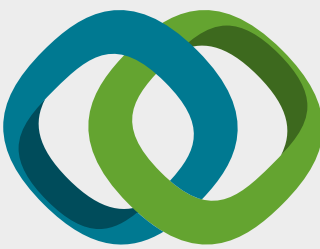

\section{Hindawi}

Submit your manuscripts at

www.hindawi.com
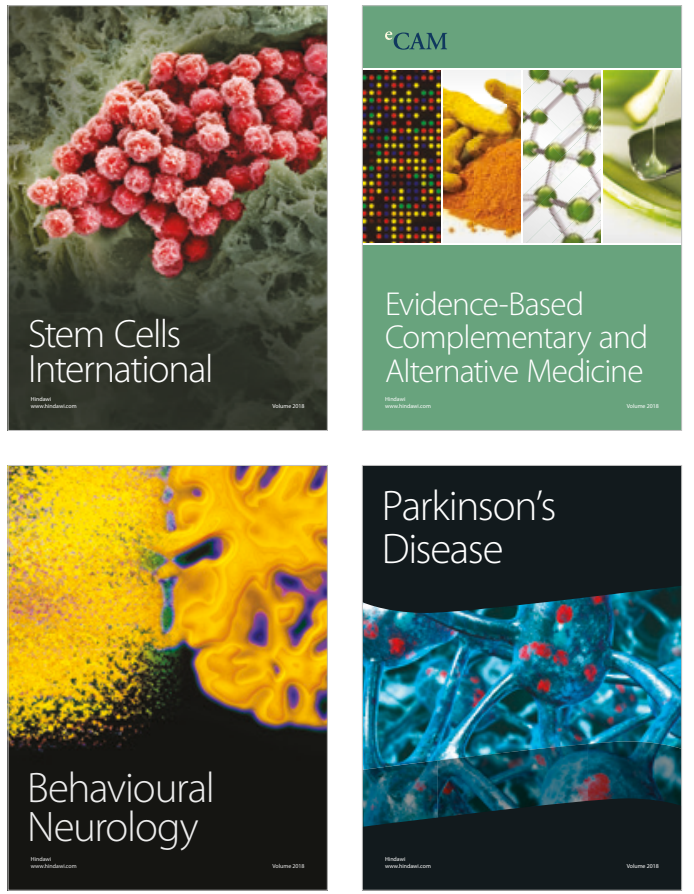

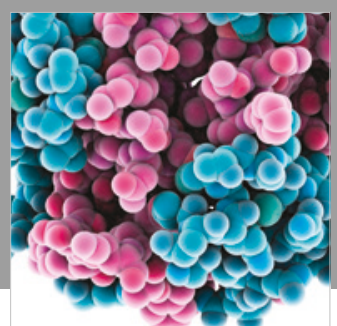

ournal of

Diabetes Research

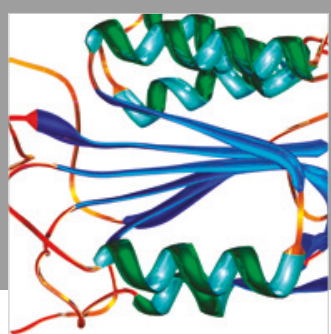

Disease Markers
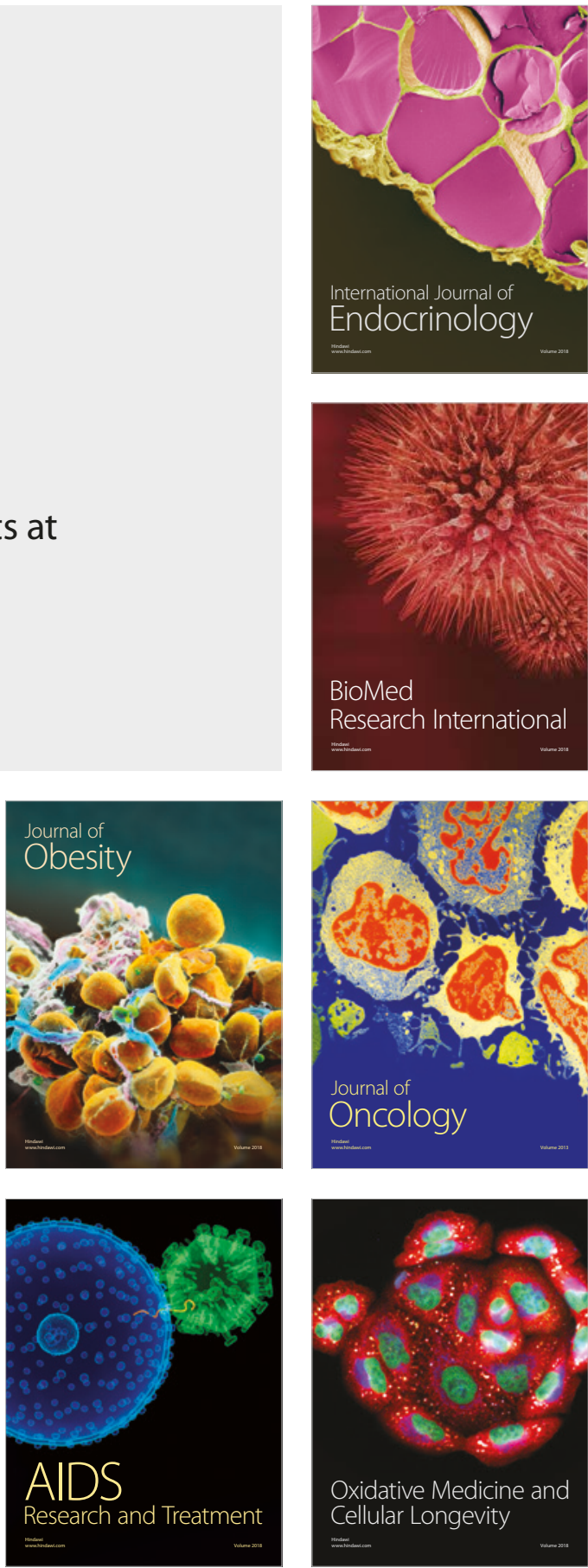